\title{
PRESENCIA FAMILIAR DURANTE LA REANIMACIÓN CARDIOPULMONAR: LA MIRADA DE ENFERMEROS Y FAMILIARES
}

\author{
FAMILY PRESENCE DURING CARDIOPULMONARY \\ RESUSCITATION: THE LOOK OF NURSES AND FAMILY
}

\author{
Marisol Silva Dall'Orso* \\ Patricia JARA CONCHA ${ }^{* *}$
}

\begin{abstract}
RESUMEN
A partir del cuestionamiento de cómo es la entrega de cuidados al paciente crítico y su familia y de cómo enfrenta el enfermero reanimador la reanimación cardiopulmonar acompañada de familiares, este estudio tuvo como objetivos describir el significado que tiene para el familiar presenciar la reanimación de un ser querido y para los enfermeros el hacer reanimación en presencia de familiares y relacionar las características sociodemográficas de familiares y de enfermeros con la valoración que hacen de la presencia de la familia durante la reanimación cardiopulmonar en el ámbito prehospitalario. Estudio exploratorio con enfoque mixto cualitativo y cuantitativo que utilizó como orientación teórica el interaccionismo simbólico. Participaron en entrevistas en profundidad y respondiendo a un instrumento de Presencia Familiar, familiares que presenciaron las maniobras de reanimación de su ser querido en su domicilio, junto a enfermeros reanimadores que trabajan en los móviles de avanzada del Servicio de Atención Médica de Urgencias (SAMU) de la Región del Bío-Bío. Los resultados permiten establecer que tanto familiares como enfermeros valoran la Presencia Familiar como beneficiosa. Respecto de la relación entre las características sociodemográficas de familiares y enfermeros y la valoración que hacen de la presencia de la familia durante la reanimación cardiopulmonar, sólo se encontró una relación significativa entre la variable edad de los enfermeros reanimadores y la valoración de la presencia familiar. Así también, los resultados permitieron identificar grandes temas que emergieron del análisis de los relatos de los familiares (experiencia impactante, comunicación simbólica y experiencia tranquilizadora) y de los enfermeros (práctica insegura, experiencia empática y práctica necesaria), los que contribuyen a fortalecer la humanización del cuidado del paciente crítico, valorar más el programa ministerial del hospital amigo y fundamentan la necesidad de formular protocolos que mejoren la calidad en salud.
\end{abstract}

Palabras clave: Resucitación cardiopulmonar, atención prehospitalaria, familia, presencia familiar.

\begin{abstract}
From questioning about how the delivery of care to critically ill patients and their families and how the nurse rescuer confronts CPR accompanied by relatives, this study aimed to describe the meaning that witness the resuscitation of loved relatives has for the family as well as for nurses doing resuscitation in the presence of family. It also relate family socio-demographic characteristics and nurses as well, with the family presence assessment during pre-hospital cardiopulmonary resuscitation setting. Exploratory study with mixed qualitative and quantitative approach, using symbolic interactionism as theoretical guidance. Relatives that witnessed resuscitation of their loved ones at home with rescuers nurses working in Bío-Bío advanced Mobile Emergency Care Service (SAMU), participated in interviews and answering to a familiar presence tool. Results indicate that both families and nurses appreciate the family presence as beneficial and in relation to socio-demographic and family presence, although there are differences, the older nurses who valued family presence as less beneficial than younger ones, turned out to be the only statistically significant difference. On the other hand, results allow
\end{abstract}

\footnotetext{
*Enfermera. Docente Depto. Enfermería. Universidad de Concepción-Chile. Email: marisilva@udec.cl
}

** Enfermera. Docente Depto. Enfermería. Universidad de Concepción-Chile. Email: pjara@udec.cl 
to identify major themes that emerged from the analysis of narratives from relatives (shocking experience, symbolic communication and comforting experience) and nurses (unsafe practice, empathic and practical experience that is required), which contribute to strengthen humanization of critical patient care, appreciate the National hospital program as friend based and substantiate the need to develop protocols that improve health quality.

Key words: Cardiopulmonary resuscitation, prehospital care, family, family presence.

Fecha recepción: 20/10/10 Fecha aceptación: 11/11/12

\section{INTRODUCCIÓN}

El Modelo Integral de Salud (1) respalda la incorporación de la familia en cada una de las acciones de salud de los distintos programas ministeriales (2). Uno de los pilares es el concepto de "Cuidado centrado en la familia", reforzando así para enfermería las bases holísticas de la práctica del cuidado (3), ya que no es sólo entregado a los pacientes, sino que al grupo familiar o redes de relaciones que tienen los pacientes.

Como políticas ministeriales para el período 2006 - 2010 en Chile, se comienza a implementar un modelo de salud integral que intenta avanzar hacia una salud más comprometida, acogedora y participativa. En este modelo se inserta el Programa del Hospital Amigo, que pretende hacer más cercana la institución de salud a los actores de ellas y a sus familiares, sin embargo ha sido compleja su ejecución, debido a que significa un cambio en el paradigma al interior de las unidades asistenciales (4).

El desarrollo de investigaciones acerca de la presencia familiar, definida como la presencia y participación de uno o más miembros de la familia en el área del cuidado del paciente, en un lugar que le/s permita el contacto visual y/o físico con el paciente durante las maniobras de reanimación (5) en el ámbito prehospitalario, comienza en la década de los ochenta desde el ámbito hospitalario. Desde entonces ha tenido un desarrollo creciente, explorando las repercusiones psicológicas negativas a corto y mediano plazo de los familiares que la viven (6), de las argumentaciones que tendrían los miembros del 'staff' de salud a favor o en contra, como de los temores reportados por los familiares (7), junto con el rol que pudiera desempeñar dentro de la práctica clínica del manejo del paciente gravemente enfermo (8).

Desde la aparición en el año 1995 del Servicio de Atención Médica de Urgencias (SAMU) en Chile, se lleva a los domicilios y a la calle un sistema de atención con procedimientos y técnicas de reanimación que antes eran exclusivos del medio intrahospitalario. La participación de enfermería en este campo es fundamental, ya que presta cuidados de calidad, desarrollados bajo protocolos avalados internacionalmente, que demandan un proceder singular según las condiciones del paciente y no descuida a los familiares que están expectantes y deseosos que se les responda al sinnúmero de interrogantes que les surgen en base al por qué de lo que están viendo y de lo que le podría significar a futuro. Los enfermeros reanimadores que se desempeñan en esta área han sabido responder exitosamente a esta demanda, enmarcando su actuar dentro del cuidado humanizado (9), realizando procedimientos con altos estándares de calidad.

La presencia familiar durante las maniobras de reanimación, sin embargo es un tema de controversia. La idea de que un miembro de la familia esté presente durante la reanimación cardiopulmonar es apoyada (10) y respaldada por diversas organizaciones internacionales, como la Emergency Nurses Association (ENA), la American Heart Asso- 
ciation (AHA) y la European Ressucitation Council $(11,12)$.

En el ámbito prehospitalario, los enfermeros reanimadores se ven sometidos a estrés, presión y demostración de su experiencia, en entornos desafiantes, caracterizados por espacios estrechos, iluminación deficiente, falta de apoyo clínico y presencia de familiares deseosos que se reestablezca lo antes posible la condición de su ser amado. Todas estas características de la práctica de enfermería prehospitalaria harían pensar que es extremadamente difícil y casi insostenible la ejecución de maniobras de calidad, sin embargo, es posible a pesar de lo determinante que puede llegar a ser la realización de maniobras de reanimación cardiopulmonar acompañada por familiares.

A su vez, por la inexistencia de estudios chilenos que abordan esta temática, este estudio tuvo como objetivos describir el significado que tiene para el familiar presenciar la reanimación de un ser querido y para los enfermeros el hacer reanimación en presencia de familiares y relacionar las características sociodemográficas de familiares y de enfermeros con la valoración que hacen de la presencia de la familia durante la reanimación cardiopulmonar en el ámbito prehospitalario. Con ello se busca mostrar la experiencia de 33 enfermeros reanimadores de la Región del Bío-Bío con respecto a la realización de las maniobras de reanimación acompañada por familiares, junto a la experiencia de 30 familiares que estuvieron presentes durante las maniobras de reanimación de su ser querido, atendidos por los profesionales del SAMU, como también comprender las relaciones que establecen y la forma de cómo se construyen las percepciones individuales, en base a gestos, roles y acciones realizadas por ellos para darle significado a la experiencia.

Se recurrió a la Teoría psicológica y social del Interaccionismo Simbólico, la que ayudó a comprender el proceso de creación y asignación de significados, y a describir el resultado de esta experiencia relacional para contribuir a la comprensión del cuidado de enfermería a nivel de la atención prehospitalaria. De esta manera, el abordaje cualitativo permitió apreciar el dinamismo de las emociones que se viven durante la experiencia (13).

El Interaccionismo Simbólico se consideró adecuado para orientar el proceso del pensamiento, debido a que la comprensión de los significados de la experiencia relacional que se produce entre los familiares y los enfermeros reanimadores del SAMU se construyen a partir de la interacción social que se produce entre ellos y de los intensos momentos que viven. Como esencia del Interaccionismo (14) se dan 3 premisas: 1) los seres humanos actúan frente a las cosas en base a los significados que éstas tienen para ellos, 2) el significado emerge de la interacción social que se tiene con los otros y 3 ) los significados son usados y transformados, por medio de procesos interpretativos que la persona emplea, mientras se ve enfrentado a diferentes situaciones. Así, los significados que los eventos tienen para los familiares y enfermeros reanimadores se basan fundamentalmente en la interacción emanada de las acciones ejecutadas entre ellos y el ambiente que rodea a las maniobras de reanimación.

\section{METODOLOGÍA}

Estudio exploratorio con enfoque mixto cualitativo y cuantitativo, retrospectivo. Se decidió de tipo retrospectivo debido a lo sensible de esta temática, sobre todo en el grupo de los familiares y así permitirles que depuraran la experiencia y lograr que desde una mirada más lejana y objetiva pudieran comunicar lo vivido por ellos en ese difícil momento. El abordaje pretendió describir lo que se vive en una reanimación acompañada por familiares y el de vivir una reanimación de un ser querido, como de comprender el significado de estas vivencias. Se utilizó un instrumento basado en el cuestionario de Presencia Familiar 
de la Emergency Nurses Association (ENA) para obtener los resultados cuantitativos de valoración de la presencia familiar como beneficiosa o menos beneficiosa (7) y la perspectiva de la investigación cualitativa para hacer visibles las necesidades de los familiares y revelar la realidad de la práctica enfermero.

En los dos grupos de estudio la muestra fue por conveniencia, para lo cual se efectuó una revisión de los folios de atención de los móviles de avanzada SAMU de Concepción y Talcahuano entre los años 2007 y 2008, agrupando los que correspondían a atención de pacientes por sufrir paro cardiorrespiratorio; se visitaron los domicilios, entre marzo 2009 a mayo 2010, se les informó del estudio (participación voluntaria, sin beneficio directo ni riesgos, a confidencialidad y anonimato, libertad para retirarse y uso de los datos) y se solicitó a los familiares que hubieran presenciado las maniobras y que quisieran participar firmaran el consentimiento informado, resultando la muestra en 30 familiares, lo que correspondió al 47\% del universo. La muestra de los 33 enfermeros reanimadores correspondió a los que trabajaban en el móvil de avanzada SAMU de la Región del BíoBío Chile (específicamente de las ciudades de Cañete, Chillán, Concepción, Los Ángeles y Talcahuano) durante los años 2007 a 2009 y que de forma voluntaria aceptaron responder el instrumento y acceder a la entrevista, correspondiendo al $61 \%$ del universo, las que se realizaron desde marzo a octubre de 2009.

La investigación se realizó luego de la aprobación del Comité de Ética de la Facultad de Medicina de la Universidad de Concepción y de la autorización correspondiente de los jefes del SAMU de los establecimientos de salud a los cuales pertenecían los enfermeros.

La realización de las entrevistas a los familiares se abordó a través de las siguientes preguntas: ¿Cómo vivió su experiencia durante las maniobras de reanimación?, ¿qué es lo más significativo que recuerda? y ¿cómo describiría su participación en la reanimación? En los enfermeros reanimadores la entrevista giró en torno a la pregunta ¿cómo describirías tu experiencia de realizar maniobras de reanimación en un domicilio contando con la presencia de los familiares?

La recolección de los datos se realizó de manera individualizada, aplicando y grabando en una primera fase las entrevistas abiertas y en profundidad, con una duración entre 30 a $60 \mathrm{~min}$, realizadas en los domicilios de los familiares y en los lugares de descanso (residencias) de los enfermeros. De esta forma se obtuvieron los datos sociodemográficos y los relatos, que fueron analizados tras la transcripción fiel de las cintas y se procedió al análisis temático. Posteriormente se aplicó el instrumento de Presencia Familiar basado en el cuestionario de la ENA (7), que mide la presencia familiar a través de la valoración de acuerdo en 15 ítemes en el caso de los familiares y 14 para enfermeros, con 5 opciones de respuesta: Muy de acuerdo: 5, De acuerdo: 4, Indeciso: 3, En desacuerdo: 2, Muy en desacuerdo:1; con un máximo de puntuación por respuesta de 5 puntos y un mínimo de 1 punto. La interpretación se realiza en base al total de la suma de ítemes, considerando la presencia familiar beneficiosa (en los familiares una media mayor o igual a 45 puntos y en los enfermeros una media mayor o igual a 42 puntos) o menos beneficiosa (en los familiares una media menor a 45 puntos y en los enfermeros una media menor a 42 puntos). Este instrumento fue sometido a pruebas de confiabilidad (alfa de Cronbach de 0,802 para el instrumento de los enfermeros reanimadores y de 0,768 para el instrumento de los familiares) y validez de contenido, siendo sometido a un panel de expertos formado por dos enfermeros/as, un médico, una psicóloga y una persona no vinculada al área de la salud, que revisaron la relevancia, validez, pertinencia y claridad de los contenidos del Instrumento de Presencia Familiar y de la Entrevista, tanto de Enfermeros reanimadores como de Familiares. Ellos realizaron sugerencias en cuanto a la formulación de las afirmaciones, léxico y orden de las materias; 
y para la validez de constructo se consideró adecuado realizar pruebas de convergencia y divergencia. Por lo que se aplicó al grupo piloto, formado por 6 Enfermeros y 6 Familiares, los cuestionarios elegidos para dichas pruebas. Se escogió para confrontar con el Instrumento de Presencia Familiar de Enfermeros Reanimadores y realizar la convergencia el Cuestionario Caring Behaviors Inventory (CBI). Para la prueba de divergencia del Instrumento de Presencia Familiar en Enfermeros Reanimadores se buscó un instrumento que midiera aspectos negativos del cuidado, por lo que se seleccionó el Aggression Questionnaire (AQ), ambos presentaron sólo correlaciones positivas, pero no significativas, pero que para efectos de este estudio se consideraron adecuadas debido al tamaño de la muestra. Para hacer convergencia con el Instrumento de Presencia Familiar de los Familiares, se utilizó la adaptación del Cuestionario de Satisfacción Usuaria del Ministerio de Salud (MINSAL), obteniendo una significancia bilateral $\mathrm{p}<0.013$, por lo que se puede decir que el Instrumento de Presencia Familiar de los Familiares es altamente predictivo para medir la satisfacción usuaria. Para hacer la divergencia se escogió el Cuestionario de Incertidumbre frente a la enfermedad de Mishel, que sólo mostró una correlación positiva, pero no significativa, también explica- do por el tamaño de la muestra.

Para el procesamiento de las entrevistas se utilizó el Análisis Temático según Van Manen (15), y así tras la transcripción fiel de los relatos, las primeras lecturas permitieron identificar ideas generales y luego, al analizar línea por línea y frase por frase, se logró encontrar los elementos descriptores más específicos de la Presencia Familiar.

Adicionalmente, se utilizó análisis descriptivo porcentual y $\mathrm{t}$ de Student para el análisis de relación de las medias de la valoración de la presencia familiar con características sociodemográficas del familiar y sociodemográficas y profesionales del enfermero reanimador.

\section{RESULTADOS}

Se expone primero con el uso de figuras los hallazgos cualitativos y luego con el uso de tablas se dan a conocer los resultados cuantitativos.

En síntesis, de las entrevistas en profundidad se develaron 3 temas principales que emergieron de la Presencia Familiar en los familiares, los que fueron: una experiencia impactante, comunicación simbólica y una práctica tranquilizadora (Figura 1).

Figura 1. Temas principales de la Presencia Familiar como vivencia de los familiares.

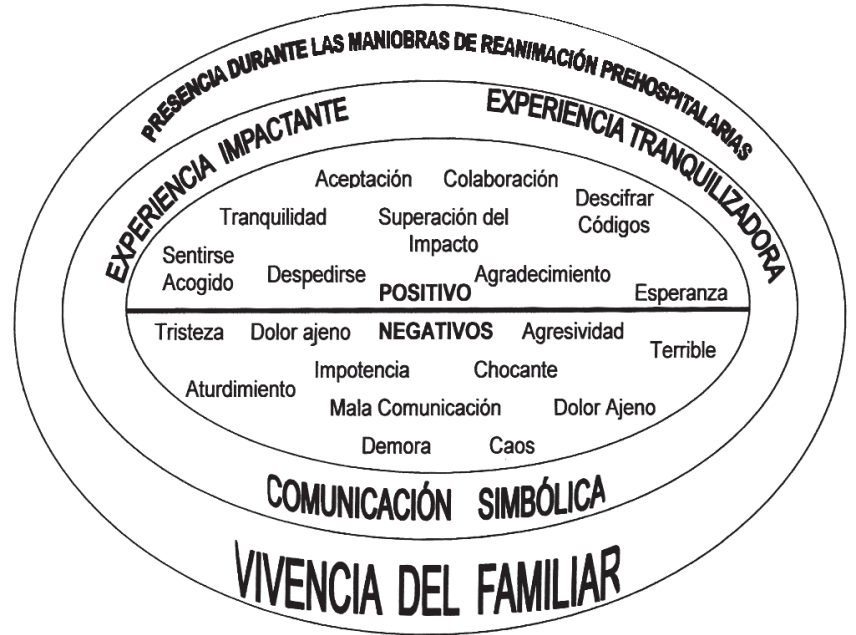


Experiencia Impactante: para los familiares resulta ser una experiencia impactante porque algunos vieron cómo súbitamente su familiar, hasta ese momento sano, sufrió paro cardiorrespiratorio y su vida dependía ahora del éxito de las maniobras. Para otros, también fue impactante, porque a pesar de que acompañaron todo el proceso de una enfermedad terminal de su ser querido, tampoco estaban preparados para despedirse. Además porque tras el impacto de darse cuenta de la gravedad del hecho, al momento que ven que llega la ayuda y a su ser querido lo someten a maniobras agresivas en "el suelo" y enigmáticas para algunos, o conocidas por otros, pero que ahora se la estaban efectuando a su familiar, fue sin duda impactante. Sin embargo, la mayoría de los familiares tratan de no ser fuentes de preocupación para el equipo que viene a ayudarlos y tratan de quedarse expectantes a los movimientos y requerimientos de los enfermeros del SAMU. Esto se puede identificar en los siguientes relatos:

Familiar 1: “...Somos tan poca cosa [sonríe nerviosamente], porque te pescan y...bueno, yo sé que eso es lo que tienen que hacer, no van a estar con cuidado sacándole la ropa, sería imposible, pero no valimos nada... porque te pescan y te hacen todas las cosas así. Es chocante en ese momento y uno debe estar preparada que así tiene que ser no más".

Familiar 10: "Muy, muy fuerte... todo rápido, pero fue una cosa que SE DESARMÓ TODO, esa pieza [indica con la mano una habitación], quedó toda desarmada... Llegó la ambulancia, sacaron todo, pusieron máquinas y seguramente para que yo no me diera cuenta de que él ya se había ido, me dijeron HAGA ESTO... que hiciera 1, 2 y 3 y que le apretara, con esa cosita que andan trayendo, todas esas cosas de máquinas que le colocan, todo".

Comunicación Simbólica: para ellos, el establecimiento de las relaciones se basa en los gestos y actitudes que pueden interpretar de los enfermeros reanimadores, ya que la mayoría se queja de una escasa comunicación verbal con ellos, "entendible" según ellos mismos, porque deben instaurar lo antes posible maniobras de reanimación, por eso intentan descubrir el significado "del idioma" del equipo, como también descifrar cada una de las acciones, miradas o movimientos que realizan los enfermeros reanimadores buscando señales que los ayuden a captar la esencia de lo que están viviendo. Lo que se ejemplifica con los siguientes relatos:

Familiar 4: “...lo dejaron allá en esa pieza, en el suelo... sacaron un aparato grande, como una plancha grande y se lo pusieron aquí [llevándose las manos al pecho] y empezó a saltar... entonces me dio... como dolor, mal genio, pena, me dio rabia también, porque en algún momento, como que ya no quería que le hicieran nada. Como que uno ya sabía que estaba muerto, me daba la impresión que él estaba sufriendo".

Familiar 1: "Yo estaba observando no más y veía lo que le decía el médico al otro, y que contaban los minutos ... yo miraba no más, porque no podía interrumpir, porque al final iba a estorbar a la persona que estaba haciendo todo lo posible, porque yo vi que le estaba haciendo todo lo posible. Porque si a lo mejor yo no hubiera visto, a lo mejor hubiera quedado con dudas". "Si me hubieran dicho, mire sujete aquí, yo lo habría hecho, pero en ese momento no fue necesario porque habian 3 personas".

Familiar 8 (164-167): "Bueno ellos a medida que hacían las cosas iban como hablando... dentro de su idioma, resulta que ese día teníamos también a una prima que también es enfermera, entonces ella era como el traductor en ese aspecto".

Práctica Tranquilizadora: paradójicamente para los familiares resulta ser una experiencia tranquilizadora, ya que de los relatos emerge la seguridad que sintieron del equipo 
que vino a "ayudarlos". La cohesión del equipo y la premura con que actuaron, como relatan ellos, significó que se sintieran más tranquilos al ver que ya se le estaba "haciendo algo". Todos agradecen y/o reconocen el esfuerzo desplegado por el equipo para poder revivir a su ser querido, dicen que el profesionalismo que vieron les ayudó a sentirse más confiados. Lo que refleja que, a pesar de los angustiantes, amargos e imborrables recuerdos que tienen, salvo 3 familiares de los 30 , todos volverían nuevamente a presenciar las maniobras de reanimación de un ser querido. Evidenciado por los siguientes relatos:

Familiar 2: "La enfermera que andaba... fue súper amable y no sé, me dio como tranquilidad, la forma de actuar de ella, me dio tranquilidad a mí, de que se estaban haciendo las cosas bien. Porque de repente..., si algún funcionario es medio hostil o tiene otra forma... uno cree que las cosas se están haciendo mal y que... todo mal".

Familiar 9: "La atención fue buena, sí buena, porque actuaron como debían actuar, rápido, oportuno, claro y dando esperanza. Dando una esperanza de que podrían volverla a la vida, todo lo que se hizo... se hizo en pos de eso. Tratar de reanimarla y volverla a la vida, pero no fue..."

El análisis de las entrevistas en profundidad de los enfermeros reanimadores resultó en 3 temas principales, que fue la Presencia Familiar como una: práctica insegura, una experiencia empática y una práctica necesaria (Figura 2).

Figura 2. Temas principales de la Presencia Familiar como vivencia de los Enfermeros Reanimadores.

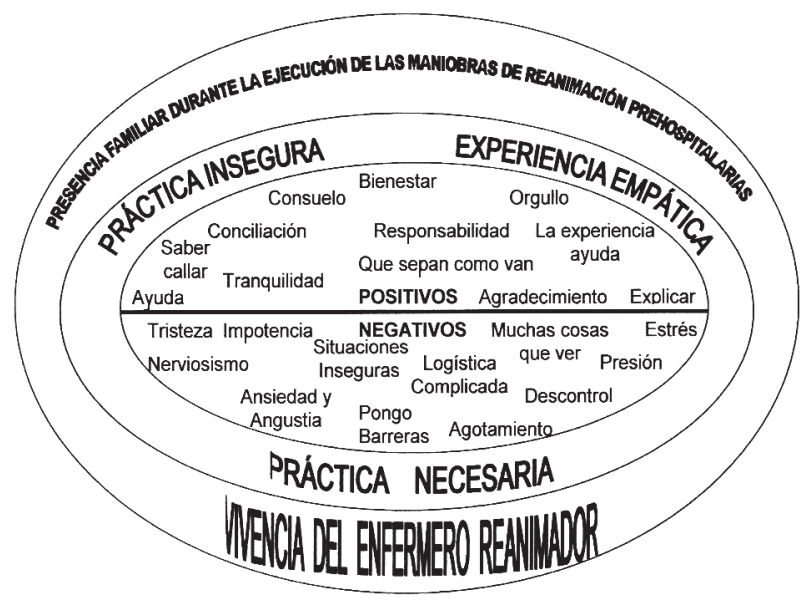

Práctica Insegura: en general para todos, el hecho de abordar las reanimaciones en los domicilios es insegura porque la atención debe dirigirse a múltiples focos; el paciente, la atención diligente, el entorno y los familiares, además de sentirse constantemente evaluados teniendo que demostrar confianza y profesionalismo, se suma el descontrol familiar, que puede llegar a interferir con las maniobras. La inseguridad física, mencionada anteriormente, se superpone a la inseguridad emocional, de ver el sufrimiento ajeno constantemente, junto al involucrarse de una u otra forma de la situación de paro que repercute en ellos, lo que se refleja en los siguientes relatos: 
Enfermero 9: “...El compromiso emocional y toda la desesperación de la gente influye y entorpece las maniobras, porque se te tiran encima, no lo quieren soltar, no te dan espacio para trabajar, lloran, patalean, se te desmayan encima".

Enfermero 7: "Yo como no mostrar... no soy como muy... [y traza una barrera imaginaria con la mano derecha, desde su cabeza hasta su pecho] yo creo que es una forma de defenderme de ellos, pero no defenderme de lo que me puedan hacer, sino que defenderme de lo que a mí me provoca la sensación de que se muera la gente..."

Experiencia Empática: todos los enfermeros reanimadores creen que es bueno tener a los familiares presentes durante las maniobras de reanimación. Comprenden como lógicas las reacciones que tienen los familiares al verse enfrentados a la reanimación de su ser querido, las aceptan y no las ven como entorpecedoras, sino que más bien las modulan para hacerlas cooperativas. Tratan de acogerlos porque también les gustaría que al estar en su posición a ellos los acogieran. Lo que se puede mostrar a través de los siguientes relatos:

Enfermero 11: "Uno está entrando a 'su casa', donde hay un familiar de ellos que está falleciendo, entonces...Todo tipo de reacciones yo creo que son 'entendibles', si yo me pusiera en el lugar de ellos... en realidad no sé cuál sería mi reacción".

Enfermero 13: "En relación a cómo los afecta... generalizando, yo creo que la tendencia es más positiva que negativa, porque les ayuda, si el paciente fallece, enfrentan mejor el proceso de duelo, de ver que hicieron todas las acciones, de ver que se trató de salvar a esta persona y si la persona muere y queda ahí,... de despedirse 'en el mismo momento', en el momento que los creyentes piensan que el alma se desprende del cuerpo y están ahí para despedirlo, o de preocuparse en última instancia de esta persona en la Tierra, de poder abrigarlo, de darle besitos, tocarlo... que es importante para muchas personas, ese contacto físico final y que en otras instancias no se da".

Práctica Necesaria: para los enfermeros reanimadores se hace necesaria ya que se acude a 'sus' domicilios y ellos son los expertos en delinear o mostrar las mejores vías de acceso hacia el paciente o lugares más confortables para que se establezca la reanimación, también pueden aportar datos relevantes sobre los antecedentes médicos de su ser querido, que puede influir favorablemente en el éxito de las maniobras. También en algunas ocasiones y si el familiar está relativamente tranquilo, puede cooperar con las mismas técnicas que suponen las maniobras de reanimación. Además que al ir viendo las maniobras y cuando se van comunicando con ellos, los van preparando para los resultados desfavorables. Lo que se describe en los siguientes relatos:

Enfermero 12: "Es útil y necesario, porque faltan manos para que sujeten un suero o conecten un alargador, porque se acabó la batería del monitor, o que despejen la pieza, el lugar de salida, despejen de muebles, que busquen las pastillas en caso de intoxicaciones..."

Enfermero 3: "Al principio pensaba... por mucho tiempo que esto no era bueno, pero la experiencia y las situaciones que he vivido... se encargaron de demostrarme que era necesario. Y yo creo que no es sólo necesario para el $S A M U$, sino que debiera ser necesario en una asistencia pública, porque... lo más probable es que ese paciente... Estadísticamente va a morir, entonces ése es el último momento para la persona, para estar con su familiar y para demostrarle que se hizo lo que se tenía que hacer y no tenemos nada que esconder... si tú haces bien las cosas no tienes nada que esconder. No tienes que cerrar las cortinas, ¿por qué?. Si no estás haciendo nada malo... entonces yo creo 
que de repente, cerrar las puertas, es esconder lo que estás haciendo... y todos sabemos de repente por qué pasa..."

En resumen, la vivencia de los familiares al estar presentes durante la ejecución de las maniobras de reanimación y de los enfermeros reanimadores al efectuar maniobras de reanimación teniendo a los familiares presentes, se concentra en ambos grupos en tres grandes temas, que se expresan de ma- nera dinámica durante el transcurso de las maniobras, a través de diferentes emociones, conductas y comportamientos que se equilibran entre significar una experiencia negativa, repercutiendo gravemente en el bienestar familiar y profesional o suscita una experiencia positiva llevando a la resiliencia del enfermero reanimador y del familiar.

En cuanto a los resultados cuantitativos, la Tabla 1 muestra a los 30 familiares participantes, la mayoría son mujeres (70\%), con

Tabla 1. Distribución de variables sociodemográficas de los familiares

\begin{tabular}{|c|c|c|c|}
\hline \multicolumn{4}{|c|}{ FAMILIARES } \\
\hline Variables & & Frec & $\%$ \\
\hline \multirow{2}{*}{ Sexo } & Femenino & 21 & 70 \\
\hline & Masculino & 9 & 30 \\
\hline \multirow{4}{*}{ Edad } & Menos 30 años & 6 & 20 \\
\hline & $30-36$ años & 10 & 33,1 \\
\hline & $37-62$ años & 11 & 36,6 \\
\hline & 63 y más años & 3 & 9,9 \\
\hline \multirow{2}{*}{ Religión } & Con & 27 & 90 \\
\hline & Sin & 3 & 10 \\
\hline \multirow{3}{*}{ Nivel de instrucción } & Superior & 11 & 36,6 \\
\hline & Media & 13 & 43,3 \\
\hline & Básica & 6 & 20 \\
\hline \multirow{4}{*}{ Actividad ocupacional } & Dueña Casa & 10 & 33,3 \\
\hline & Personal Salud & 2 & 6,6 \\
\hline & Actividad Rem. & 10 & 33,3 \\
\hline & Actividad No rem. & 8 & 26,7 \\
\hline
\end{tabular}

edades entre los 18 a 69 años, casi la totalidad se identifica con alguna creencia religiosa (90\%), su nivel de instrucción reveló que gran parte tenía nivel medio y superior $(80 \%)$ y casi el $60 \%$ de ellos realizaban actividades no remuneradas.
Los familiares valoraron la Presencia Familiar como beneficiosa, siendo las medias totales por sobre 45 puntos en el Instrumento de Presencia Familiar.

En la Tabla 2 se presentan los resultados correspondientes a la aplicación del Instru-

Tabla 2. Distribución de variables sociodemográficas de los familiares según Medias de Presencia Familiar (2009).

\begin{tabular}{|l|l|c|c|}
\hline \multicolumn{4}{|c|}{ FAMILIARES } \\
\hline Variables & \multicolumn{2}{|c|}{ Significancia } \\
\hline \multirow{3}{*}{ Sexo } & Femenino & 51,8 & \multirow{2}{*}{ Tc: $0,008 \quad \mathrm{p}=0,994$} \\
\cline { 2 - 3 } & Masculino & 51,77 & \\
\multirow{4}{*}{ Edad } & Fenos 30 años $0,36 \quad \mathrm{p}=0,782$ \\
\cline { 2 - 3 } & $30-36$ años & 53,66 & \\
\cline { 2 - 3 } & $37-62$ años & 49,9 & \\
\cline { 2 - 3 } & 63 y más años & 54 & \\
\hline
\end{tabular}


Continuación Tabla 2.

\begin{tabular}{|c|c|c|c|}
\hline \multirow{2}{*}{ Religión } & Con & 51,03 & \multirow{2}{*}{ Tc: $-1,22 \quad p=0,230$} \\
\hline & $\operatorname{Sin}$ & 58,66 & \\
\hline \multirow{3}{*}{ Nivel de instrucción } & Superior & 54,36 & \multirow{3}{*}{ Fc: $1,420 \quad p=0,259$} \\
\hline & Media & 48,23 & \\
\hline & Básica & 54,83 & \\
\hline \multirow{4}{*}{ Actividad ocupacional } & Dueña Casa & 52,7 & \multirow{4}{*}{ Fc: 1,303} \\
\hline & Personal Salud & 41 & \\
\hline & Actividad Rem. & 49,9 & \\
\hline & Actividad No rem. & 55,75 & \\
\hline
\end{tabular}

mento de Presencia Familiar de los familiares y la relación con variables sociodemográficas y profesionales. La relación de la presencia familiar con características sociodemográficas mostró que no existen diferencias en las medias obtenidas tanto en sexo, edad como en nivel de instrucción del familiar, ya que todos la consideraron igualmente beneficiosa. En relación a religión, quienes declararon no tener una religión específica valoraron la presencia familiar como más beneficiosa que los que declararon tener una religión, pero la diferencia no fue significativa. En relación a la actividad ocupacional, quienes declararon ser personal de salud valoraron la presencia familiar como menos beneficiosa, aunque no se obtuvieron diferencias significativas.

La Tabla 3 muestra a los Enfermeros Reanimadores participantes que fueron 33, la mayoría eran varones (61\%), el mayor

Tabla 3. Distribución de variables sociodemográficas de los enfermeros reanimadores.

\begin{tabular}{|l|l|r|c|}
\hline \multicolumn{4}{|c|}{ ENFERMEROS REANIMADORES } \\
\hline Variables & & Frec & $\%$ \\
\hline \multirow{3}{*}{ Sexo } & Femenino & 13 & 39,4 \\
\cline { 2 - 4 } & Masculino & 20 & 60,6 \\
\hline \multirow{3}{*}{ Edad } & Menos 30 años & 9 & 27,3 \\
\cline { 2 - 4 } & $30-39$ años & 22 & 66,7 \\
\cline { 2 - 4 } & 40 y más años & 2 & 6,1 \\
\hline \multirow{3}{*}{ Años exp. profesión } & Con & 26 & 78,8 \\
\cline { 2 - 4 } & Sin & 7 & 21,2 \\
\hline \multirow{3}{*}{ Años reanimal } & Menos 5 años & 8 & 24,2 \\
\cline { 2 - 4 } & $5-10$ años & 7 & 21,21 \\
\cline { 2 - 4 } & Más de 10 años & 18 & 54,54 \\
\hline & Menos 5 años & 12 & 36,36 \\
\cline { 2 - 4 } & $5-10$ años & 6 & 18,18 \\
\cline { 2 - 4 } & Más de 10 años & 15 & 45,45 \\
\hline
\end{tabular}

porcentaje con edades entre 30 a 39 años (67\%) se identificaron con alguna creencia religiosa en un $79 \%$ y la mayoría llevaba más de 10 años de experiencia profesional (54\%) y como reanimador del SAMU, correspondiendo el mayor porcentaje al grupo que tenía más de 10 años de experiencia (45\%).
Este grupo valoró la Presencia Familiar como beneficiosa, siendo la media total sobre 42 puntos en el Instrumento de Presencia Familiar.

Los resultados correspondientes a la aplicación del Instrumento de Presencia Familiar en enfermeros reanimadores y la relación con 
variables sociodemográficas y profesionales (Tabla 4) mostró que si bien las enfermeras valoran como más beneficiosa la presencia familiar que los enfermeros, no existe diferencia significativa, tampoco hay diferencia con la religión ni con los años de experiencia profesional. Sin embargo la edad del enfermero marca la diferencia en las medias obtenidas, mostrando que el enfermero de más edad percibe como menos beneficiosa la presencia familiar que aquel que es más joven, con una diferencia altamente significativa.

Tabla 4. Distribución de variables sociodemográficas de enfermeros SAMU según Media de Presencia Familiar (2009).

\begin{tabular}{|c|c|c|c|c|}
\hline \multicolumn{5}{|c|}{ ENFERMEROS REANIMADORES } \\
\hline Variables & & Media Presencia Fam & \multicolumn{2}{|c|}{ Significancia } \\
\hline \multirow{2}{*}{ Sexo } & Femenino & 46,61 & \multirow{2}{*}{ Tc: 0,589} & \multirow{2}{*}{$\mathrm{p}=0,560$} \\
\hline & Masculino & 45,35 & & \\
\hline \multirow{3}{*}{ Edad } & Menos 30 años & 44,66 & \multirow{3}{*}{ Fc: 10,25} & \multirow{3}{*}{$\mathrm{p}=0,000$} \\
\hline & $30-39$ años & 47,59 & & \\
\hline & 40 y más años & 32 & & \\
\hline \multirow{2}{*}{ Religión } & Con & 45,61 & \multirow{2}{*}{ Tc: $-0,427$} & \multirow{2}{*}{$\mathrm{p}=0,672$} \\
\hline & Sin & 46,71 & & \\
\hline \multirow{3}{*}{ Años exp. profesional } & Menos 5 años & 44,25 & \multirow{3}{*}{ Fc: 1,605} & \multirow{3}{*}{$\mathrm{p}=0,218$} \\
\hline & $5-10$ años & 49,28 & & \\
\hline & Más de 10 años & 45,22 & & \\
\hline \multirow{3}{*}{ Años reanimador SAMU } & Menos 5 años & 47 & \multirow{3}{*}{ Fc: 0,655} & \multirow{3}{*}{$\mathrm{p}=0,527$} \\
\hline & $5-10$ años & 46,83 & & \\
\hline & Más de 10 años & 44,53 & & \\
\hline
\end{tabular}

En el análisis por ítem del Instrumento de Presencia Familiar de los familiares (Ta$y$, por lo tanto, hubo mayor variabilidad de respuestas fue el 4: "En algún momento debla 5), el ítem que obtuvo la menor media seó retirarse del lugar y/o habitación” (media

Tabla 5. Distribución de Medias por Ítem del Instrumento de Presencia Familiar del familiar (2009-2010).

\begin{tabular}{|c|c|c|c|c|}
\hline & Item & $\mathrm{N}$ & Media & D.S. \\
\hline 1 & $\begin{array}{l}\text { La Presencia Familiar durante la reanimación es beneficiosa para los } \\
\text { miembros sobrevivientes. }\end{array}$ & 30 & 36.333 & 140.156 \\
\hline 2 & $\begin{array}{l}\text { La posibilidad de separarlo de su ser querido, durante la reanimación, le } \\
\text { generó más estrés y tensión emocional. }\end{array}$ & 30 & 38.333 & 146.413 \\
\hline 3 & $\begin{array}{l}\text { La visualización de las maniobras y procedimientos invasivos, le provocó } \\
\text { ansiedad y respuestas emocionales agresivas. }\end{array}$ & 30 & 39.000 & 160.495 \\
\hline 4 & En algún momento deseó retirarse del lugar y/o habitación. & 30 & 23.667 & 175.152 \\
\hline 5 & $\begin{array}{l}\text { El estar presente durante la reanimación, le sirvió para entender lo que } \\
\text { estaba sucediendo con su ser querido. }\end{array}$ & 30 & 46.667 & 0.75810 \\
\hline 6 & $\begin{array}{l}\text { El haber presenciado y participado durante la reanimación, le ayudó a } \\
\text { despedirse de su ser querido. }\end{array}$ & 30 & 34.667 & 165.536 \\
\hline 7 & $\begin{array}{l}\text { El presenciar la reanimación de su ser querido, le sirvió para afrontar los } \\
\text { resultados de ésta. }\end{array}$ & 30 & 43.333 & 129.544 \\
\hline
\end{tabular}


Continuación Tabla 5.

\begin{tabular}{|c|l|c|c|c|}
\hline 8 & $\begin{array}{l}\text { El que se le haya permitido estar presente durante las maniobras de } \\
\text { reanimación de su ser querido, lo acercó más a los miembros del equipo } \\
\text { de salud. }\end{array}$ & 30 & 37.000 & 141.787 \\
\hline 9 & $\begin{array}{l}\text { Debe ser un derecho establecido, la posibilidad de que los familiares estén } \\
\text { presentes durante las maniobras de reanimación de su ser querido. }\end{array}$ & 30 & 41.000 & 142.272 \\
\hline 10 & $\begin{array}{l}\text { Durante la visualización de las maniobras, considera que debe existir un } \\
\text { miembro del equipo de salud apoyándolo a usted exclusivamente. }\end{array}$ & 30 & 47.000 & 0.91539 \\
\hline 11 & $\begin{array}{l}\text { Los miembros del equipo de salud deben siempre ofrecer a los familiares } \\
\text { estar presentes durante las maniobras de reanimación. }\end{array}$ & 30 & 37.000 & 126.355 \\
\hline 12 & $\begin{array}{l}\text { Es más estresante para el equipo de salud tener a miembros de la familia } \\
\text { presente durante las maniobras de reanimación. }\end{array}$ & 30 & 39.333 & 133.735 \\
\hline 13 & $\begin{array}{l}\text { Los miembros del equipo de salud actúan diferente al tener a los } \\
\text { familiares presentes durante las maniobras de reanimación. }\end{array}$ & 30 & 33.667 & 142.595 \\
\hline 14 & $\begin{array}{l}\text { Los miembros del equipo de salud lo acogieron y/o apoyaron en ese } \\
\text { momento. }\end{array}$ & 30 & 34.667 & 175.643 \\
\hline 15 & $\begin{array}{l}\text { Si se le diera la posibilidad de volver a presenciar las maniobras de } \\
\text { reanimación de un ser querido, ¿volvería a hacerlo? }\end{array}$ & 30 & 42.333 & 140.647 \\
\hline & \multicolumn{1}{|c|}{ Total } & & 57.4 & \\
\hline
\end{tabular}

2.36). Se observa que las medias más altas se encontraron en los siguientes ítemes: Ítem 5: "El estar presente durante la reanimación, le sirvió para entender lo que estaba sucediendo con su ser querido" (media 4.66) Ítem 10: "Durante la visualización de las maniobras, considera que debe existir un miembro del equipo de salud apoyándolo a usted exclusivamente" (media 4.70).

En el análisis por ítem del Instrumento de Presencia Familiar de los enfermeros reanimadores (Tabla 6), se observa que las medias

Tabla 6. Distribución de Medias por Ítem del Instrumento de Presencia Familiar del Enfermero Reanimador (2009).

\begin{tabular}{|c|l|c|c|c|}
\hline & Item & $\mathrm{N}$ & Media & D.S. \\
\hline 1 & $\begin{array}{l}\text { La Presencia Familiar durante la reanimación es beneficiosa para los } \\
\text { miembros sobrevivientes. }\end{array}$ & 33 & 4.18 & 1.044 \\
\hline 2 & $\begin{array}{l}\text { El separar a los familiares de su ser querido durante la reanimación, les } \\
\text { genera más estrés y tensión emocional a ellos. }\end{array}$ & 33 & 4.30 & 0.951 \\
\hline 3 & $\begin{array}{l}\text { El dejar que los familiares presencien la reanimación es un derecho de la } \\
\text { familia. }\end{array}$ & 33 & 4.15 & 1.064 \\
\hline 4 & $\begin{array}{l}\text { La Presencia Familiar durante la reanimación, le facilita a los familiares el } \\
\text { proceso de pérdida y/o duelo en el hogar. }\end{array}$ & 33 & 4.42 & 0.902 \\
\hline 5 & $\begin{array}{l}\text { Los familiares que presencian la reanimación de su ser querido pueden } \\
\text { perder el control e interferir con las maniobras. }\end{array}$ & 33 & 3.21 & 1.293 \\
\hline 6 & $\begin{array}{l}\text { Los miembros del equipo de salud se comportan distinto al tener a los } \\
\text { familiares presente durante la maniobras de reanimación cardiopulmonar. }\end{array}$ & 33 & 2.58 & 1.415 \\
\hline 7 & $\begin{array}{l}\text { La Presencia Familiar podría influir adversamente en la realización de las } \\
\text { maniobras por parte del equipo de salud. }\end{array}$ & 33 & 2.06 & 1.171 \\
\hline 8 & $\begin{array}{l}\text { Las reacciones emocionales de los familiares le causan mayor ansiedad y } \\
\text { distracción al actuar. }\end{array}$ & 33 & 2.09 & 1.100 \\
\hline
\end{tabular}


Continuación Tabla 6.

\begin{tabular}{|c|l|c|c|c|}
\hline 9 & $\begin{array}{l}\text { La Presencia Familiar durante la reanimación, provee la oportunidad de } \\
\text { educar a la familia acerca de la condición de su ser querido. }\end{array}$ & 33 & 4.12 & 1.083 \\
\hline 10 & $\begin{array}{l}\text { La formación que obtuvo durante su desarrollo profesional, le entregó } \\
\text { herramientas para manejar esta temática. }\end{array}$ & 33 & 2.88 & 1.431 \\
\hline 11 & $\begin{array}{l}\text { El tener presente a los familiares durante las maniobras de reanimación, le } \\
\text { conduce a una menor destreza en las técnicas. }\end{array}$ & 33 & 1.73 & 0.911 \\
\hline 12 & $\begin{array}{l}\text { La Presencia Familiar durante la reanimación puede interferir con la } \\
\text { enseñanza de nuevos profesionales. }\end{array}$ & 33 & 2.48 & 1.149 \\
\hline 13 & $\begin{array}{l}\text { El inadecuado liderazgo por parte del equipo de salud puede dar una } \\
\text { impresión de un tratamiento subóptimo con repercusiones médico-legales. }\end{array}$ & 33 & 3.97 & 1.015 \\
\hline 14 & $\begin{array}{l}\text { Deben existir protocolos formales que regularicen la Presencia Familiar } \\
\text { durante las maniobras de reanimación. }\end{array}$ & 33 & 3.67 & 1.051 \\
\hline & TOTAL & & 45.84 & \\
\hline
\end{tabular}

más altas se encontraron en los siguientes ítemes: Ítem 4: "La Presencia Familiar durante la reanimación, le facilita a los familiares el proceso de pérdida y/o duelo en el hogar" (media 4.42); Ítem 2: "El separar a los familiares de su ser querido durante la reanimación, les genera más estrés y tensión emocional a ellos" (media 4.30) y el Ítem 1: "La Presencia Familiar durante la reanimación es beneficiosa para los miembros sobrevivientes" (media 4.18). El ítem en los enfermeros que obtuvo, en promedio, el menor puntaje fue el 11: "El tener presente a los familiares durante las maniobras de reanimación, le conduce a una menor destreza en las técnicas" (media 1.73). Al igual que en los familiares, también existe dispersión en las respuestas, aunque ésta es más baja que en el grupo anterior, lo que indica que existe un grado mayor de consenso en las opiniones de los enfermeros reanimadores respecto a aspectos que rodean el concepto de Presencia Familiar.

\section{DISCUSIÓN Y CONCLUSIÓN}

La presencia familiar durante las maniobras de reanimación es más valorada como beneficiosa por los enfermeros que por los familiares, al igual que en lo descrito por varios autores $(5,8,16)$, sin embargo existen otros reportes que señalan $(17,18)$ que enfermeros perciben la presencia familiar con más aspectos negativos que positivos. La opinión favorable de que los familiares presencien las maniobras de reanimación de su ser querido, en los enfermeros reanimadores, se contrapone al resultado de Díaz (17), en unidades de pacientes críticos, donde a pesar de tener políticas de visitas abiertas en el momento de la reanimación preferían que los familiares no estuvieran presentes.

Respecto a la relación de la valoración de la presencia familiar durante las maniobras de reanimación con las características sociodemográficas de los familiares es interesante profundizar en el hallazgo que aquellos familiares que tenían una actividad laboral como personal de salud percibieron la presencia familiar como menos beneficiosa, que otros familiares con otras actividades ocupacionales. Es posible pensar que su experiencia en el área de salud les haga tener más información y ver con menos valor que los familiares estén presentes durante las maniobras de reanimación cardiopulmonar de un ser querido. A pesar de lo anterior, las entrevistas en profundidad mostraron como muy positiva la oportunidad de estar presentes durante la reanimación de un familiar. Al igual que lo encontrado en un estudio en el ámbito prehospitalario español (15), la mitad de los fa- 
miliares está a favor de la presencia familiar, sin embargo hay muchos indecisos respecto a lo favorable que sea el presenciar las maniobras de reanimación, dado principalmente a la gran carga emocional y fuerte experiencia que les significó esta práctica, según relatan los mismos familiares.

Se detectó la misma variedad de conductas y comportamientos en los familiares, que los encontrados por Morse y Pooler (13) en Sala de Trauma hospitalaria. Este resultado coincide con un estudio dirigido a describir las actitudes de los profesionales de salas de emergencia frente a la presencia de familiares durante la reanimación, que encontraron que un $64 \%$ opinaba que la presencia de la familia podría inhibir la experticia en la realización de las maniobras durante la resucitación, el $71.1 \%$ creía que podría prolongarse el tiempo de resucitación y el $72 \%$ pensaba que podría aumentar el estrés del personal de salud (19).

Ello coincide también con hallazgos que muestran que los familiares se sienten capaces y están dispuestos a presenciar las maniobras en un ser querido, junto con que creen que es su derecho (20) y en relación al rol tranquilizador de presenciar las maniobras, al entender lo que sucede con su ser querido y afrontar mejor los resultados de éstas (8). Ambos aspectos son elementos fundamentales que manifiestan los familiares, los que deben ser escuchados para brindar cuidados humanizados y consistentes con sus necesidades de atención.

La necesidad de que un miembro del equipo apoye a los familiares durante las maniobras de reanimación se ve fundamentada en los lineamientos establecidos por Nibert y Ondrejka (21) y Wagner (19), en donde pequeñas intervenciones influirían favorablemente en la disminución del estrés familiar.

En relación a las características sociodemográficas y profesionales del enfermero reanimador, aunque no hay diferencias significativas, resultó que el que tiene más años de experiencia como reanimador de SAMU valora como menos beneficiosa la presencia familiar que el que tiene menos años de experiencia. Este no ha sido un hallazgo en otros estudios, pero coincide con la edad del enfermero que sí resultó estadísticamente significativa, mostrando que el enfermero de más edad percibe como menos beneficiosa la presencia familiar que aquel que es más joven. Este hallazgo no se relaciona con la valoración por ítem del instrumento de Presencia Familiar que obtiene las medias más altas en los Ítem 4: "La Presencia Familiar durante la reanimación, le facilita a los familiares el proceso de pérdida y/o duelo en el hogar" y 2: "El separar a los familiares de su ser querido durante la reanimación, les genera más estrés y tensión emocional a ellos".

Llama la atención que la mayoría de los enfermeros consideren que en los momentos de reanimación, el estrés, descontrol y tensión emocional de los familiares no provoque interferencia ni dificultad en la labor del enfermero y valoren la presencia familiar como beneficiosa, reducidora de estrés, facilitadora del proceso de duelo y un derecho de la familia. Esta conducta está en coherencia con la opinión que existe en los enfermeros reanimadores, de que la Presencia Familiar no les hace errar en los procedimientos ya que el hecho de habituarse con la presencia de los familiares durante las maniobras de reanimación les hace perder ese asombro por el familiar y fijar su atención en realizar de la mejor manera su trabajo.

Durante las entrevistas, los enfermeros reanimadores manifestaron que eran insuficientes las herramientas entregadas en su formación de pregrado, respecto a como abordar y manejar esta práctica con la familia.

Falta explorar la conducta en grupos de enfermeros que trabajen exclusivamente con distintos grupos etáreos, para indagar si se establece lo reportado en estudios previos en donde los enfermeros/as pediátricos están más a favor de esta práctica $(22,23)$, a diferencia de lo obtenido por Halm (24) y Mason 
(25), quienes describen que uno de los obstáculos para no permitir la presencia familiar es el probable aumento de demandas legales, situación que en el grupo de enfermeros no fue lo mencionado como argumento.

De este modo se concluye que la presencia familiar durante las maniobras de reanimación cardiopulmonar de un ser querido, medida a través de un instrumento de presencia familiar en el ámbito prehospitalario, resultó ser valorada como beneficiosa tanto por los familiares como por los enfermeros reanimadores. En general, la relación con variables sociodemográficas muestra que hay diferencias en las medias totales, siendo la edad del enfermero reanimador donde se observa que esta diferencia es significativa con respecto a las demás variables, estableciéndose que a mayor edad del enfermero reanimador (40 y más años), la valoración de la presencia familiar es menos beneficiosa. En los familiares, si bien todas las variables permiten establecer que la presencia familiar es medida como beneficiosa, ninguna variable es significativa estadísticamente.

El análisis de los relatos de la vivencia de la Presencia Familiar desde el punto de vista de los familiares dio origen a 3 temas fundamentales: la Presencia Familiar como una experiencia impactante, como una comunicación simbólica y una práctica tranquilizadora. Es posible relacionar estos 3 temas con los conceptos que se desprenden de los ítemes que contiene el Instrumento de Presencia Familiar, como: reducidor de estrés, facilitador del duelo familiar, desencadenante de la ansiedad familiar y la Presencia Familiar como entorpecedora de las maniobras. Estos conceptos permiten nuevos elementos al análisis de los dos grupos de estudio.

Por otra parte, el análisis de los relatos de la vivencia de la Presencia Familiar durante las maniobras de reanimación, desde el punto de vista de los/as enfermeros/as reanimadores, da origen a 3 temas fundamentales: la Presencia Familiar como una práctica insegura, una experiencia empática y una prácti- ca necesaria, lo que se puede relacionar con los conceptos de la presencia familiar vista como un derecho del familiar, como práctica entorpecedora y como facilitador del proceso de duelo, según el Instrumento de Presencia Familiar aplicado, lo que permite dar consistencia a la importancia de la exploración de esta práctica de manera mixta para la mayor comprensión de la entrega de cuidados al final de la vida y para que se establezca la Presencia Familiar como derecho normado. Promover la implementación de que los familiares presencien las reanimaciones de sus seres queridos, que un miembro del equipo de salud apoye y esté satisfaciendo las necesidades de los familiares.

El abordaje mixto utilizado permitió comprender y extraer los componentes esenciales de cómo se vive la Presencia Familiar durante la reanimación, en el ámbito prehospitalario, lo que permitió conceptualizar en categorías temáticas dicha práctica para que sirvan de base en investigaciones futuras, en otras áreas del desarrollo de enfermería.

Los relatos emanados, cargados de contenidos, son prueba de una mayor toma de conciencia de los familiares que están presenciando las maniobras de reanimación de su ser querido, para comenzar a involucrarlos en la entrega de cuidados, abordando holísticamente a la persona en riesgo vital y a sus familiares, dentro del contexto del cuidado humanizado.

Se espera que este estudio estimule nuevos abordajes de la presencia familiar, en las diferentes áreas de desarrollo de enfermería $y$, al mismo tiempo, contribuya a abrir un campo inexplorado de la investigación tradicional chilena, que hace posible investigar la reanimación cardiopulmonar desde la perspectiva de quienes la están viviendo.

La comprensión de la Presencia Familiar en el contexto de la reanimación contribuye a ampliar los conceptos que sustentan el Programa Hospital Amigo del Ministerio de Salud de Chile. Los resultados son un aporte a la implementación de protocolos de acuer- 
do a la normativa vigente.

Es así como un estudio europeo (17) menciona que dentro de los profesionales de enfermería, los que más desarrollan y mejor valoran esta práctica son los que trabajan en unidades pediátricas, se hace necesario realizar una comparación entre valoración de la Presencia Familiar que hacen los enfermeros prehospitalarios y los enfermeros intrahospitalarios tanto de unidades de pacientes críticos de adultos como pediátricos, para tener un posicionamiento de enfermería y protocolizar la presencia familiar a nivel nacional.

\section{REFERENCIAS}

1. Ministerio de Salud. Orientaciones para la programación en red [Internet]. Chile: Gobierno de Chile Ministerio de Salud; 2008 [citado 10 noviembre 2012]. Disponible en: http://www.redsalud.gov.cl

2. Consejo Internacional de Enfermeras. Cuidar a la familia - Las enfermeras dispuestas siempre a ayudarte [Internet]. Ginebra: Consejo Internacional de Enfermeras; 2002 [citado 11 enero 2012]. Disponible en: http://www.icn.ch/images/stories/documents/publications/ind/ indkit2002_01sp.pdf

3. Consejo Internacional de Enfermeras. Código Deontológico del CIE para la profesión de enfermería [Internet]. Ginebra: Consejo Internacional de Enfermeras; 2006 [citado 10 noviembre 2012]. Disponible en: http://www.icn.ch/images/stories/documents/about/icncode spanish.pdf

4. Ministerio de Salud. Avances en Salud 2007 [Internet]. Chile: Ministerio de Salud; 2007 [citado 10 noviembre 2012]. Disponible en: http://www.minsal.gob.cl

5. Eichhorn DJ, Meyers TA, Guzzetta CE, Clark AP, Klein JD, Talieaferro E, et al. Family presence during invasive procedures and resuscitation: hearing the voice of the patient. Am J Nurs. 2001; 101(5): 4855.

6. Robinson SM, Mackenzie-Ross S, Campbell-Hewson GL, Egleston CV, Prevost AT. Psychological effect of witnessed resuscitation on bereaved relatives. Lancet. 1998; 352: 614-17.

7. MacLean SL, Guzzetta CE, White C, Fontaine D, Eichhorn DJ et al. Family presence during cardiopulmonary resuscitation and invasive procedures: practices of critical care and emergency nurses. Am J Crit Care. 2003; 12(3): 246-57.

8. Fein JA, Ganesh J, Alphern ER. Medical staff attitudes toward family presence during pediatric procedures. Pediatric Emerg Care. 2004; 20(4): 224-27.

9. Ceballos P. Desde los ámbitos de enfermería, analizando el cuidado humanizado. Cienc. enferm. 2010; XVI (1): 31-5.

10. Project Team of the Resuscitation Council (UK). Should Relatives Witness Resuscitation? [Internet]. Londres; Resuscitation Council (UK) 1996 [citado 10 noviembre 2012]. Disponible en: http:// www.resus.org.uk/pages/witness.pdf

11. American Heart Association (AHA). Ethical Aspects of CPR and ECC. Circulation. 2000; 102: 112-21.

12. Baskett PJF, Steen PA, Bossaert L. European Resuscitation Council Guidelines for Resuscitation 2005. Section 8 The ethics of resuscitation and end-of-life decisions. Resuscitation. 2005; 67(Suppl 1): S171-80.

13. Morse JM, Pooler Ch. Patient-FamilyNurse Interactions in the Trauma-Resuscitation Room. Am J Crit Care. 2002; 11(3): 240-49.

14. De la Cuesta C. La teoría fundamentada como herramienta de análisis. Cultura de los Cuidados. 2006; X (20): 136-40.

15. Van Manen M. Researching Lived Experience. 2nd ed. Canadá: The Althouse Press; 1997.

16. De la Flor B, Castelo I, Andetxaga I, Peláez M, Gil E, Aguirre A. Presencia Fami- 
liar durante maniobras de reanimación: estudio en el ámbito extrahospitalario en la comunidad autónoma vasca. Emergencias. 2006, 18(3): 135-40.

17. Díaz H, Véliz M, Mestre J. Encuesta sobre la presencia familiar durante los procederes invasivos y la reanimación. REMI [Internet]. 2006 [citado 11 agosto 2007]; 6(4): A45. Disponible en: http://remi. uninet.edu/2006/04/REMIA045.htm

18. Walker W. Accident and emergency staff opinion on the effects of family presence during adult resuscitation: critical literature review. J Adv Nurs. 2008; 61(4): 34862.

19. Wagner JM. Lived experience of critically ill patient's family members during cardiopulmonary resuscitation. Am J Crit Care. 2004; 13(5): 416-20.

20. Duran CR, Oman KS, Abel JJ, Koziel VM, Szymanski D. Attitudes toward and beliefs about Family Presence: A survey of healthcare providers, patients' families, and patients. Am J Crit Care. 2007; 16 (3): $270-82$.

21. Nibert L, Ondrejka D. Family Presence during pediatric resuscitation: an integrative review for evidence-based practice. J Ped Nurs. 2005; 20(2): 145-147.

22. Bradford KK, Kost S, Selbst SM, Renwick AE, Pratt A. Family Member Presence for Procedures: The Resident's Perspective. Ambul Pediatr. 2005; 5(5): 294-97.

23. Fulbrook P., Latour J. M. y Albarran J. W. Paediatric critical care nurses' attitudes and experiences of parental presence during cardiopulmonary resuscitation: a European survey. Int J Nurs Stud. 2007; 44(7): 1238-249.

24. Halm MA. Family presence during resuscitation: A Critical review of the literature. Am J Crit Care. 2005; 14(6): 494-511.

25. Mason D. Family Presence: Evidence versus Tradition. Am J Crit Care. 2003; 12(3): 190-92. 